\title{
Search for Neutrino Point Sources with the AMANDA Telescope
}

\author{
Othmane Bouhali* for the AMANDA collaboration \\ IIHE, Universit Libre de Bruxelles, Science Faculty CP230 \\ B-1050 Brussels, Belgium \\ E-mail: "bouhali@hep.iihe.ac.bè
}

ABSTRACT: We present results from the search of extraterrestrial point sources of high energy neutrinos in the Northern sky with the AMANDA telescope located at the South Pole.

\section{Introduction}

The search for sources of high energy neutrinos is one of the central missions of the Antarctic Muon And Neutrino Detector Array (AMANDA). The origin of cosmic ray acceleration to the most extreme energies is still not known. Cosmic rays are thought to be accelerated in the shock fronts of galactic and extragalactic objects like supernovae remnants, active galactic nuclei and gamma ray bursts [i]. Accelerated high energy protons will collide with the ambient gas and radiation surrounding the acceleration region, or with matter or radiation intervening between the source and the Earth. This leads to the production of neutrinos. The detection of these neutrinos will help in understanding the origin of cosmic rays acceleration and the physical processes at work in the most energetic objects of the Universe.

\section{The AMANDA Telescope}

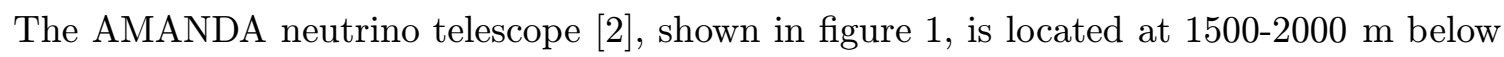
the surface of the Antarctic ice sheet at the geographic South Pole. With this specific location, AMANDA is looking at the whole Northern sky. Neutrinos are inferred from the arrival times of Cherenkov light emitted by charged secondaries produced in neutrino interactions. The AMANDA-B10 detector, which is an earlier phase of the telescope, consists of 302 optical modules (OMs) on 10 strings.

\footnotetext{
${ }^{*}$ Speaker.
} 
Each OM comprises a photomultiplier tube with its electronics housed in a glass pressure vessel. The OMs are connected to the surface by means of dedicated electrical cables, which supply the high voltage and carry the signal from the PMTs. For each event, the arrival times and the amplitudes of OMs pulses are digitized by peak ADCs and TDCs. Several in situ light sources are used to calibrate the detector and measure the ice properties. In the austral summer 1999/2000, this detector was enlarged to a total of 19 strings with $677 \mathrm{OMs}$ to form the actual AMANDA-II telescope. One unique feature of AMANDA is that it continously measures atmospheric muons in coincidence with the South Pole Air

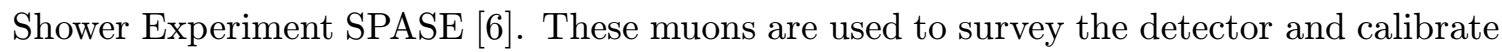

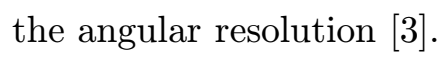

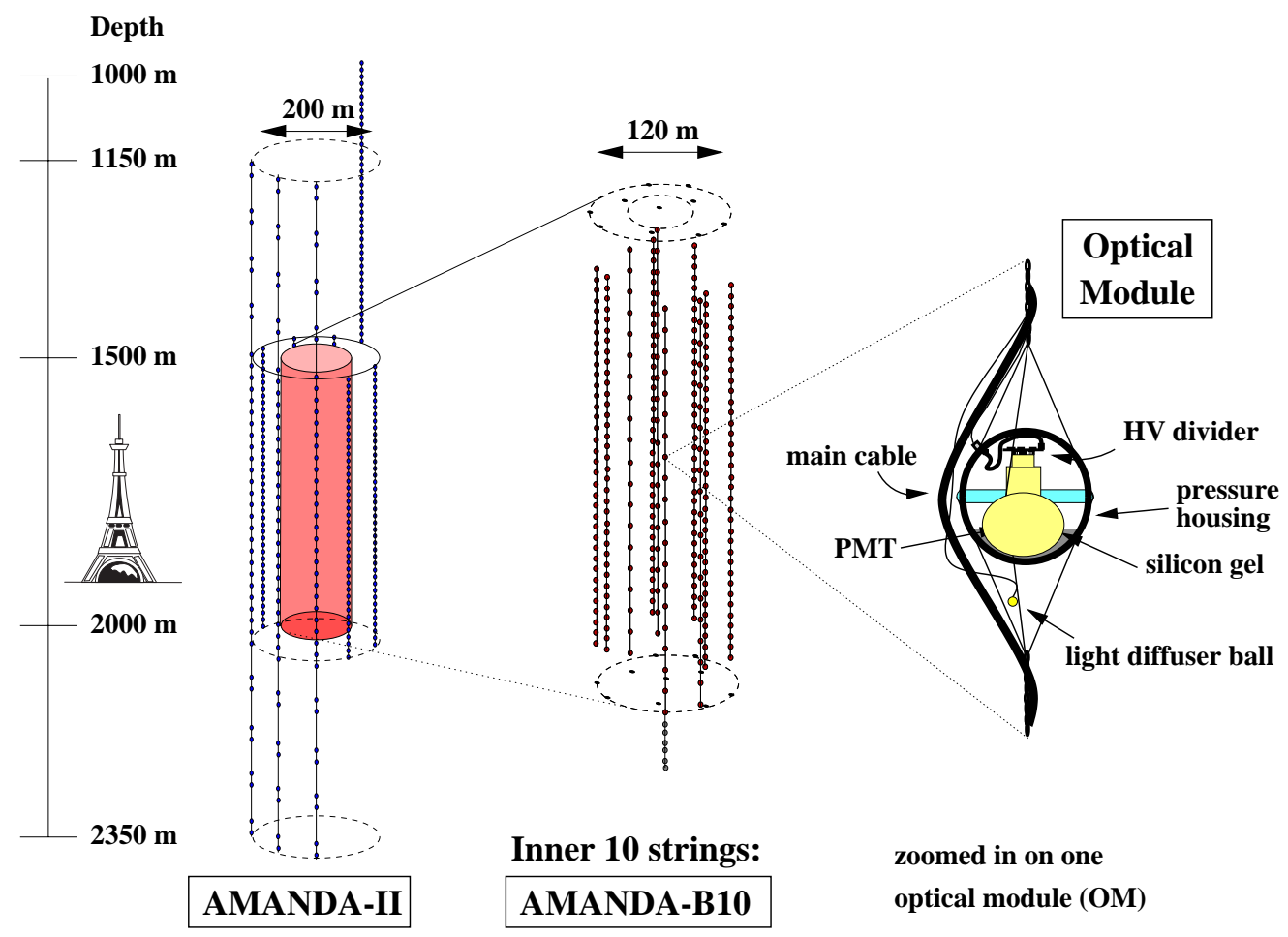

Figure 1: A schematic view of the AMANDA telescope.

\section{Data analysis and simulation}

The presence of a point source of high energy neutrinos would be revealed by a statistically significant enhancement of the number of upward traveling muons over the expected background from a particular direction. In addition, the signal of such a point source is assumed to have a hard $\left(E^{-2}\right)$ spectrum of neutrinos energies. This is a very important feature as it helps to reject poorly reconstructed atmospheric downward traveling muons reconstructed as upward traveling as well as atmospheric neutrinos, both of which have softer spectra. Data presented in this paper are from AMANDA-B10(1997 and 1999), and from AMANDA-II (2000). 
Prior to track reconstruction and event selection, runs and/or OMs exhibiting abnormal behavior are rejected. The data are also corrected for possible crosstalk induced effects. Then a number of event reconstruction techniques is applied to the data [3in]. A set of selec-

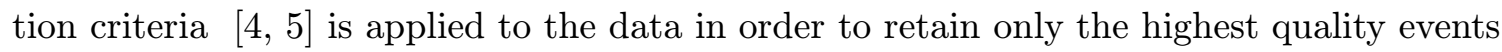
which possess topological and directional informations consistent with those expected from upward traveling neutrino-induced muons. To avoid bias in the selection criteria, the data are "blinded" by randomizing the right ascension coordinate of each event. It is only after having finalized the analysis that the correct right ascensions are restored.

A full simulation chain is performed that is used to simulate point sources, atmospheric muons and atmospheric neutrino signals [4,

Table ${ }_{1}^{1}$ ì gives some informations on the 3 data sets presented in this paper. Results for 1999 are preliminary and therefore subject to further improvements.

\section{Results and discussions}

The final stage of the data analysis procedure yields a sample of a few hundreds of upwardly reconstructed events (see table $\left.1 \underline{1}_{1}^{1}\right)$. In order to perform a quantitative search for high energy neutrino point sources, the Northern sky is divided into non-overlapping angular bins to form a grid. Point sources would be identified by the signature of an excess of events clustering in a particular direction. The optimal bin size depends on the angular resolution of the detector. Figure 2 shows the space angle resolution as obtained for AMANDA-B10 and AMANDA-II as a function of the declination. Since AMANDA-II has twice the number of OMs and a larger lever arm in the horizontal direction compared to AMANDA-B10, the angular resolution shows an improvement of almost a factor 2 .

Each bin in the grid is tested for significance of excess of events, $\xi$, given by:

$$
\xi=-\log _{10} \sum_{n=N_{0}}^{\infty} \frac{\exp ^{-\mu} \mu^{n}}{n !}
$$

where $N_{0}$ is the observed number of events and $\mu$ is the average number of background events. The grid is shifted in declination and right ascension to fully cover boundaries between the bins of the original grid. The distribution of significance shows no evidence of a source in the three data samples. This translates into an upper limit on the neutrino

\begin{tabular}{|l|l|l|l|}
\hline & $1997(\mathrm{~B} 10)$ & $\begin{array}{l}1999(\mathrm{~B} 10) \\
\text { preliminary }\end{array}$ & 2000 (AMANDA-II) \\
\hline Events at trigger level & $1.010^{9}$ & $1.310^{9}$ & $1.210^{9}$ \\
\hline Lifetime in days & 130 & 221 & 197 \\
\hline Blind analysis & - & yes & yes \\
\hline $\begin{array}{l}\text { events passing the } \\
\text { selection criteria }\end{array}$ & 815 & 621 & 699 \\
\hline
\end{tabular}

Table 1: Characteristics of the AMANDA data periods during 1997, 1999 and 2000. 


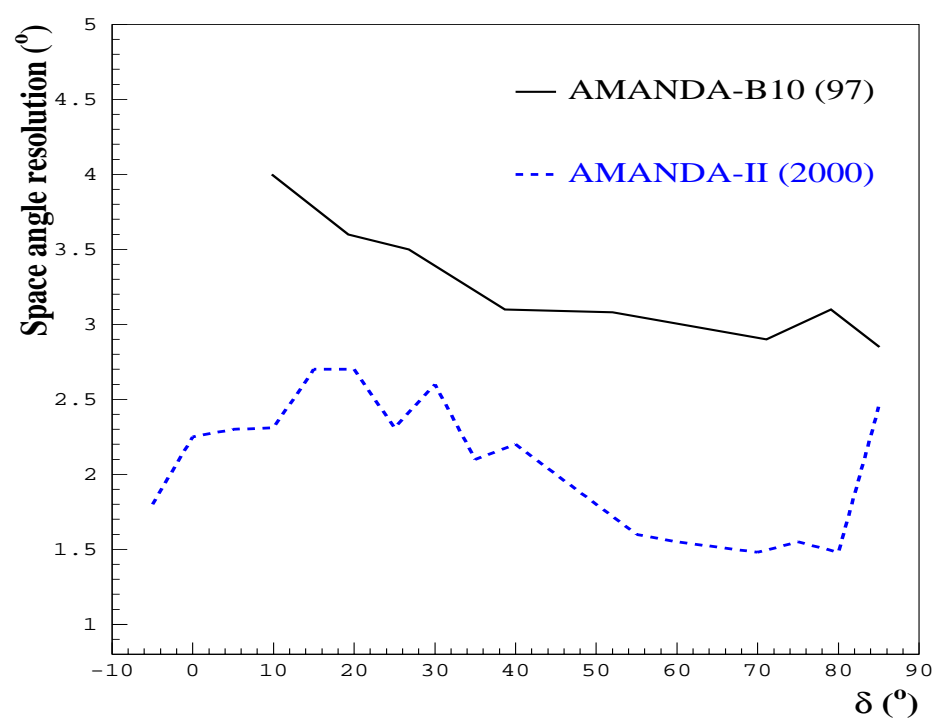

Figure 2: The dependence of the space angle resolution on the declination as obtained with AMANDA-B10 (solid line) and AMANDA-II (dashed line).

flux. This limit is given by:

$$
\phi_{\nu}(E)=\phi(E) \frac{\mu_{90}\left(n_{o b s}, n_{b}\right)}{n_{s}}
$$

where $n_{s}$ is the expected number of signal events from a simulated flux $\phi(E), n_{b}$ is the number of background events, $n_{o b s}$ is the number of observed events in the search bin, and $\mu_{90}$ is the $90 \%$ upper limit on the number of events following the unified ordering prescription of Feldman and Cousins [i]. The overall systematic uncertainty is incorporated

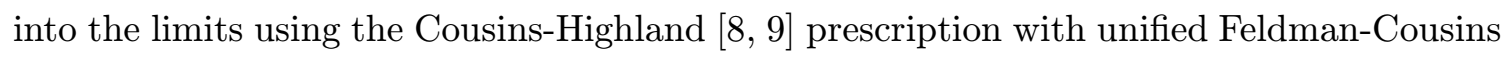
ordering [i]̄].

Figure ${ }^{13}$ 's shows the average upper limit on the neutrino flux for $E^{-2}$ spectra as obtained with AMANDA-B10 (1997 and 1999) and AMANDA-II (2000). The preliminary 99 results

\begin{tabular}{|l|l|l|l|l|l|}
\hline & & & \multicolumn{3}{|c|}{$\phi_{\nu}\left(10^{-8} \mathrm{GeVcm}^{-2} \mathrm{~s}^{-1}\right)$} \\
\hline Candidate & Dec $\left[^{\circ}\right]$ & Rad $[\mathrm{h}]$ & $1997(\mathrm{~B} 10)$ & $\begin{array}{l}1999(\mathrm{~B} 10) \\
\text { preliminary }\end{array}$ & 2000 (AMANDA-II) \\
\hline Mkn 421 & 38.2 & 11.07 & 11.2 & 3.7 & 3.5 \\
\hline Mkn 501 & 39.8 & 16.90 & 9.5 & 7.6 & 1.8 \\
\hline Cygnus X3 & 41.0 & 20.54 & 4.9 & 5.2 & 3.5 \\
\hline SS 433 & 5.0 & 19.20 & - & 1.35 & 0.7 \\
\hline Crab Nebula & 22.0 & 5.58 & 4.2 & 9.2 & 2.4 \\
\hline
\end{tabular}

Table 2: Neutrino flux limits on some selected sources in units of $10^{-8} \mathrm{GeVcm}^{-2} \mathrm{~S}^{-1}$ computed for a neutrino spectrum of $E^{-2}$ with $E>10 \mathrm{GeV}$. 


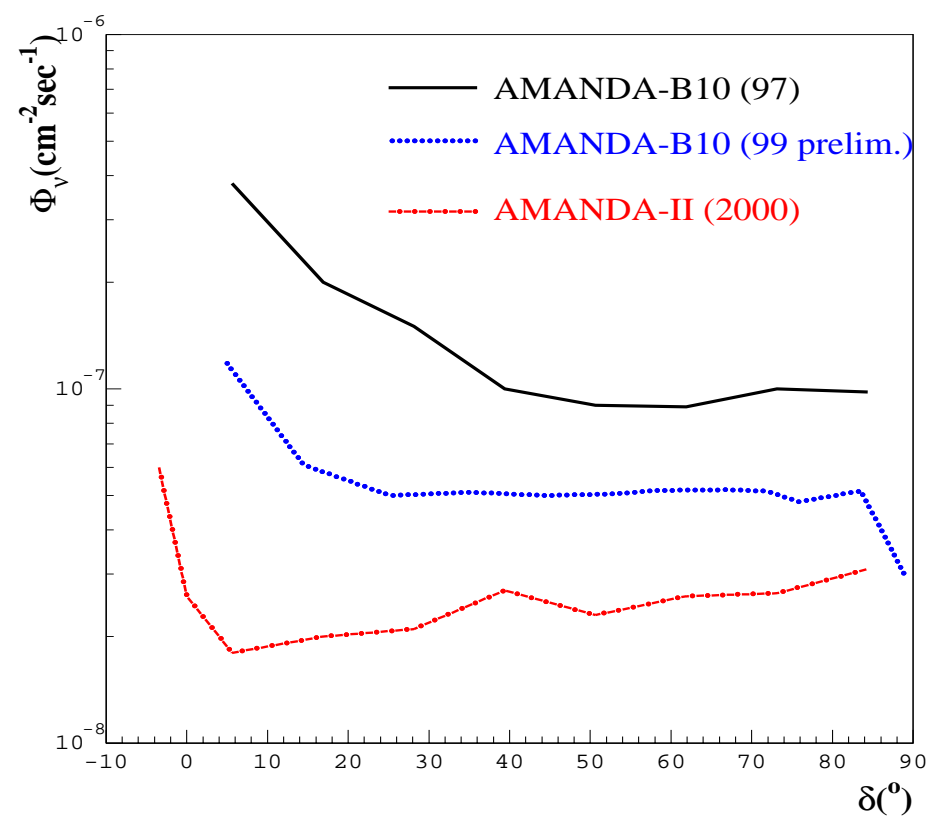

Figure 3: Average flux upper limits $(90 \% \mathrm{CL})$ for an $E^{-2}$ signal spectrum, integrated above $E_{\nu}=10 \mathrm{GeV}$. The results are shown as a function of the declination for 1997 (solid line), preliminary 1999 (dashed line) and 2000 (dashed-dotted line).

lead to a better limit compared to those obtained in 97 for two major reasons. On one hand the detector lifetime is higher as can be seen from table analysis and optimization procedures have been improved.

With AMANDA-II, the limits are much better thanks to the enlarged detector and to the powerful optimization method used [5]

In addition to the general search for a point source, a number of potential galactic and extragalactic candidate sources in the northern hemisphere are investigated by performing the significance test while centering the search bin on their sky coordinates. The resulting flux limits, for some of those sources, are presented in Table ${ }_{2}^{\prime}$ Limits are computed based on an assumed $E^{-2}$ energy spectrum. Expected numbers of neutrino induced muons for galactic microquasars have been calculated in [i] $[1]$, using source parameters estimated from radio observations. In Figure 通 the AMANDA sensitivity is compared to the 1997-averaged $\mathrm{TeV}$ gamma-ray flux of Markarian 501. This flux is corrected for intergalactic absorption by infrared photons. The comparison demonstrates that AMANDA-II has achieved the sensitivity needed to search for neutrino fluxes from $\mathrm{TeV}$ gamma-ray sources of similar strength to the intrinsic gamma-ray flux.

\section{Acknowledgments}

This research was supported by the following agencies: National Science Foundation-Office of Polar Programs, National Science Foundation-Physics Division, 


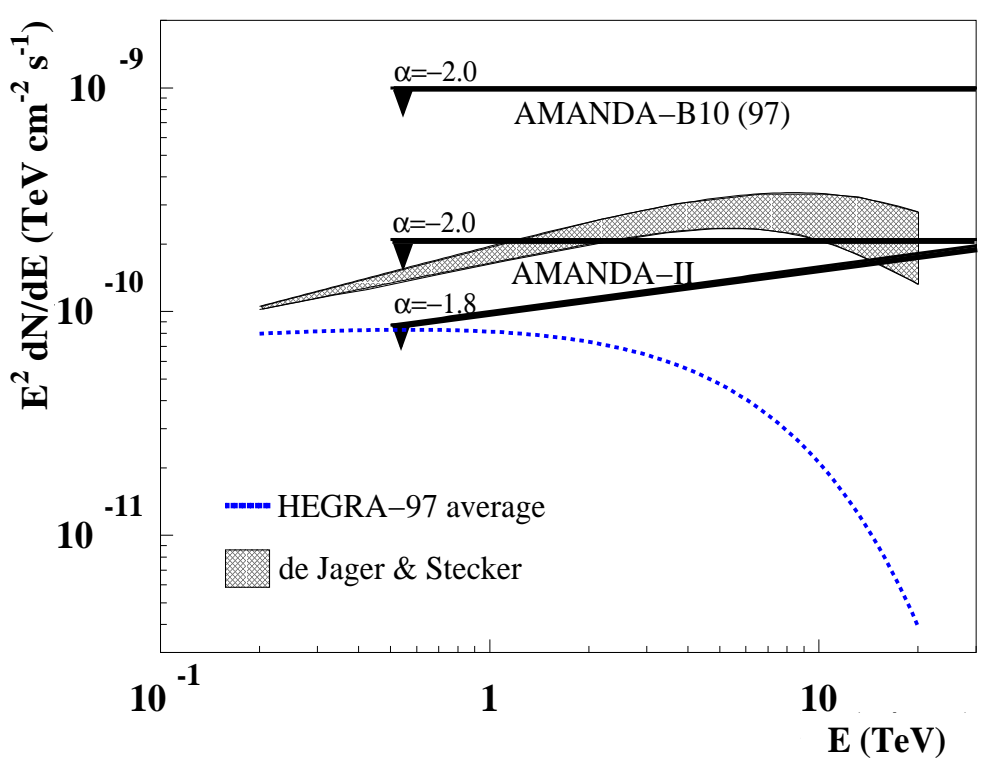

Figure 4: Comparison of the AMANDA 90\% average upper limit to the average gamma-ray flux of Markarian 501 as observed in 1997 by HEGRA [110]". Two spectral indices are shown for AMANDAII. The intrinsic source flux factor after correction by Jager and Stecker [1] $\left.{ }^{2}\right]$ is also shown.

University of Wisconsin Alumni Research Foundation, Department of Energy, and National Energy Research Scientific Computing Center (supported by the Office of Energy Research of the Department of Energy), UC-Irvine AENEAS Supercomputer Facility, USA; Swedish Research Council, Swedish Polar Research Secretariat, and Knut and Alice Wallenberg Foundation, Sweden; German Ministry for Education and Research, Deutsche Forschungsgemeinschaft (DFG), Germany; Fund for Scientific Research (FNRS-FWO), Flanders Institute to encourage scientific and technological research in industry (IWT), and Belgian Federal Office for Scientific, Technical and Cultural affairs (OSTC), Belgium. D.F.C. acknowledges the support of the NSF CAREER program.

\section{References}

[1] F. Halzen and D. Hooper, Rep. Prog. Phys. 65 (2002) 1025

[2] E. Andres et al,. 'Ástropart. Physs. 13 2000$) \overline{1}$

[3] J. Ahrens et al., paper submitted to iNucl. Instrum. Meth. A $(2003)$;

[4] J. Ahrens et al., iAstrophys.

[5] J. Ahrens et al., accepted by 'Pיhys. Rev. Lett. _ (2003) I

[6] J. Ahrens et al., accpeted by Astropart. Phys. (2003)!

[7] G. J. Feldman and R.D. Cousins

[8] R. D. Cousins and V. L. Highland, 'Nucl. Instrum. Meth. ÁĀ20

[9] J. Conrad et al., iPhys. Rev. D 67.02003$)$ 012002:

[10] F. Aharonian, Astron. Astrophys. 393 
[11] C. Distefano, D. Guetta, A. Levinson and E. Waxmann, Astrophys. J. 575

[12] O.C. de Jager and F. W. Stecker, Astrophys. J. $\mathbf{5} 6 \mathbf{6} 6(2002) 738$ 